\title{
Inhibitor of DNA-binding protein 1 knockdown arrests the growth of colorectal cancer cells and suppresses hepatic metastasis in vivo
}

\author{
XIAOLAN LAI ${ }^{1}$, JINRONG LIAO ${ }^{1}$, WANSONG LIN ${ }^{2,4}$, CHUANZHONG HUANG ${ }^{2,4}$, \\ JIEYU LI ${ }^{2,4}$, JIZHEN LIN ${ }^{3}$, QIANG CHEN ${ }^{1,3,4}$ and YUNBIN YE ${ }^{1,2,4}$ \\ ${ }^{1}$ Graduate School of Education, Fujian Medical University, Fuzhou, Fujian 350108; \\ ${ }^{2}$ Laboratory of Immuno-Oncology, Fujian Provincial Cancer Hospital, Fuzhou, Fujian 350014; \\ ${ }^{3}$ Department of Medical Oncology, Fujian Medical University Union Hospital, Fuzhou, Fujian 350001; \\ ${ }^{4}$ Fujian Key Laboratory of Translational Cancer Medicine, Fuzhou, Fujian 350014, P.R. China
}

Received January 24, 2014; Accepted February 24, 2014

DOI: $10.3892 /$ or.2014.3172

\begin{abstract}
Inhibitor of DNA-binding protein 1 (ID1) is commonly abnormally overexpressed in colorectal cancer (CRC); yet, the functional significance of ID1 in the growth and invasive properties of CRC cells remains largely unclear. The present study investigated the effects of ID1 downregulation on the cell growth and metastatic features of CRC. Using lentiviral shRNA infection, stable ID1-knockdown (KD) HCT116 and SW620 cells, human metastatic CRC cell lines, were created. In vitro, the migration/invasion capacity of the ID1-KD CRC cells was assessed by a wound healing assay. The activities of MMP2 and MMP-9 were measured by gelatin zymography. The expression of CXC chemokine receptor 4 (CXCR4), PCNA and survivin were determined by immunoblot analysis and qRT-PCR. The effects of ID1 knockdown on tumor growth and hepatic metastasis were demonstrated by a xenograft study in mice. The results showed evident decreases in proliferation, migration and invasion and an increased apoptosis rate in the ID1-KD CRC cells. Similarly, ID1 knockdown significantly decreased mRNA and protein levels of PCNA, survivin, CXCR4, MMP2 and MMP9. Overexpression of CXCR4 antagonized the negative effect on the migration and invasion abilities of the ID1-KD cells. As compared with the control, ID1 knockdown prevented tumor growth and profoundly suppressed hepatic metastasis in vivo. The present study demonstrated the significance of ID1 in colon cancer progression, and its effect on tumor invasiveness and metastatic properties may be partly dependent on CXCR4.
\end{abstract}

Correspondence to: Dr Yunbin Ye, Laboratory of ImmunoOncology, Fujian Provincial Cancer Hospital, Fujian Medical University Teaching Hospital, 420 Fu-Ma Road, Fuzhou 350014, P.R. China

E-mail: zjyunbin@189.cn

Dr Qiang Chen, Department of Oncology, Fujian Medical University Union Hospital, 29 Xin-Quan Road, Fuzhou 350001, P.R. China

E-mail: cqiang8@189.cn

Key words: ID1, colon cancer cells, proliferation, migration, hepatic metastasis, CXCR4

\section{Introduction}

As a major public health issue worldwide, colorectal cancer (CRC) is the third most common type of cancers and the second leading cause of death by cancer in the Western world (1). Development of a malignant colorectal tumor is a progressive process with a duration of several years. A series of molecular events and alterations, which involve adhesion molecules, angiogenic factors, chemotactic and growth factors, appear to be responsible for the different stages with invasion and metastatic spread (2). However, the predominant drivers contributing to CRC cell malignancy, such as their migration and invasion to the liver, remain to be determined.

Inhibitors of DNA-binding proteins (ID1, ID2, ID3 and ID4) are characterized structurally as classic basic helix-loophelix (bHLH) transcription factors but lacking a DNA-binding domain (3). The functional activation of ID proteins is initiated by forming a heterodimer with bHLH transcription factors and then blocking their DNA-binding domain, and as a result, inhibiting transcriptional activity. It is widely accepted that the accumulation of activated ID proteins function as dominant-negative regulators of bHLH transcription factors and are critical for various cellular processes in mammalian cells. ID1 was identified as a modulator of E2A to inhibit the cell cycle (4). Some ID proteins have been shown to neutralize pRB suppression on E2F-DP1 activity to potentiate $S$ phase progression $(5,6)$ and to inhibit cell differentiation in cells (7). Consistent with their functional features, ID genes are expressed abundantly in many proliferating tissues but scarcely in terminally differentiated tissues (8), suggesting their specific role in embryonic development (9).

Along with the important cellular function, dysregulation of ID expression strongly correlates with cancer progression $(10,11)$. Overexpression of IDs is a gene-expression signature in a wide variety of cancers. Therefore, IDs have been recognized as oncogenic or ontogenesis-related factors. ID1 is one of the most extensively investigated members of the ID family. Accumulating evidence has confirmed that ID1 is upregulated in several types of tumors including breast, prostate, lung, gastric, esophageal and colorectal adenocarcinoma $(12,13)$. This increase in ID1 expression appears to be 
crucial for growth grades, invasive properties and subsequently a poor clinical outcome (13-18). Evidence also indicates that expression of ID proteins, including ID1, correlates with the p53 level and the mitotic index in colorectal tumors $(19,20)$. However, our knowledge concerning the potential roles of ID1 in proliferation, migration and metastasis of colon cancer is limited (20).

In the present study, we knocked down ID1 using lentiviral shRNA to investigate the role of ID1 in CRC cell lines. We investigated whether depletion of ID1 in CRC cells is associated with inhibition of proliferation, migration/invasion and distal metastasis abilities. We also provided evidence that the function of ID1 in regulating colon cancer cell migration was partly through the chemokine receptor CXC chemokine receptor 4 (CXCR4) in the HCT116 cell line.

\section{Materials and methods}

Cell culture. Human colon cancer cell lines HCT116 and SW620 were obtained from the Shanghai Cell Bank of the Chinese Academy of Sciences. HCT116 cells were cultured in McCoy's 5A medium, and SW620 were maintained in L-15 medium supplemented with $10 \%$ fetal bovine serum (FBS). Cells were collected when at least $80 \%$ confluent for the experiments.

Gene knockdown (KD) and overexpression. All of the shId1 and control plasmids were purchased from Sigma-Aldrich Corp. The human short hairpins used to target ID1 were as follows: 5'-AGTCTCTGGTGACTAGTAG-3' (shID1-1); 5'-TGAGGCGTGAGTAACAGCC-3' (shID1-2); 5'-ACCTGCTGCTCGTCCAGCA-3' (shID1-3) and 5'-CATGTCGTAGAGCAGCACG-3' (shID1-4). A control shRNA unrelated to the human sequences was used as a negative control. The shRNA vector was co-transfected with packaging vectors pCMV-Dr82 and pCMV-VSVG at a ratio of 4:3:2 into 293T cells using Lipofectamine 2000 reagent (Invitrogen, USA). Polybrene (6 $\mu \mathrm{g} / \mathrm{ml}$; Sigma, USA) was added to the media for viral infection. For generating stable clones, the ID1-KD cells and control vector cells were selected using $1.5 \mu \mathrm{g} / \mathrm{ml}$ puromycin (Merck, Germany) for 3 weeks. The expression of ID1 was routinely detected by western blotting and quantitative real-time RT-PCR. For CXCR4 overexpression, full-length CXCR4 cDNA was cloned into a pcDNA3.0 vector using standard protocols. ID1 stable KD HCT116 cells were transfected with either a pcDNA-CXCR4 or a pcDNA3.0 empty vector using Lipofectamine 2000 reagent.

Flow cytometric analysis. Apoptosis/necrosis was determined using the PE Annexin V Apoptosis Detection Kit I (BD Biosciences), according to the manufacturer's recommendations. Samples were analyzed by flow cytometry (FACSCalibur; BD Biosciences). The experiments were performed in triplicate and repeated six times.

Proliferation assay. A total of $4 \times 10^{3}$ HCT116 or $5 \times 10^{4}$ SW620 cells/well with ID1-KD or control shRNA were seeded in 96-well plates and cultured for $48 \mathrm{~h}$. Cell proliferation was determined using the 3-(4,5-dimethylthiazol-
2-yl)-5-(3-carboxymethoxyphenyl)-2-(4-sulfophenyl)-2H-tetrazolium, inner salt (MTS) assay (Promega, Madison, WI, USA) according to the manufacturer's recommendations.

Wound healing assay and migration/invasion assays. Cells with ID1-KD shRNA or cells with control shRNA were plated in 6-well plates in duplicate at $\sim 80 \%$ confluency and allowed to grow overnight. The following day a scratch wound was made through the center of each well using a $10-\mu 1$ pipette tip. Plates were washed three times with phosphate-buffered saline (PBS), and fresh media were then added to remove any loose cells. After $48 \mathrm{~h}$, the cells were examined by light microscopy to determine resealing of the monolayer.

A Transwell migration assay was performed using $8.0-\mu \mathrm{m}$ pore insert 24-well plates (Becton-Dickinson AG, Allschwil, Switzerland). Transwell chambers were pre-coated with $1 \mu \mathrm{g} / \mathrm{ml}$ fibronectin on the underside of the membrane. A total of $1 \times 10^{5}$ HCT 116 or $5 \times 10^{5}$ SW620 cells were plated in a 24-well cell culture insert in $100 \mu \mathrm{l}$ of FBS-free media. Inserts were then placed in the well with $500 \mu \mathrm{l}$ of $20 \%$ FBS containing media. After $24 \mathrm{~h}$ (for HCT116 cells) or $48 \mathrm{~h}$ (for SW620 cells), medium and cells in the culture insert were removed. Cells at the bottom side of the insert were methanol-fixed and stained with $0.1 \%$ crystal violet. Five random fields were selected, and cells were counted at a x100 magnification to determine the average number of cells in each insert. The invasion assay was carried out in the same manner except that the $8.0-\mu \mathrm{m}$ pore size membrane insert was coated with Matrigel (BD Biosciences) that had been diluted in medium (1:5 dilution).

RNA extraction and real-time reverse transcription-PCR. Total RNA from the CRC cells was extracted using the RNeasy Mini kit, according to the manufacturer's protocol (Qiagen Inc., USA). Total cellular RNA was isolated from the xenografted tumors using TRIzol reagent (Invitrogen). One microgram of total RNA was reverse-transcribed using the Promega Reverse Transcription System A3500 (Promega). Quantitative real-time polymerase chain reaction (qRT-PCR) was run on a LightCycler Roche 480 with DyNAmo Flash SYBR-Green qPCR kit (Thermo Fisher Scientific, USA). The thermocycling program was performed according to the instrument's manual. Primers for the genes of interest are listed in Table I. For the relative quantification of the mRNA levels, 6 independent amplifications were performed for each target gene, with triplicate samples. $\beta$-actin was used as a reference gene to normalize gene expression in each sample. The relative mRNA expression levels were normalized to the level of $\beta$-actin mRNA expression (equal 1) in the corresponding samples. The data were presented numerically by the comparative $2^{-\Delta \Delta \mathrm{Ct}}$ method.

Western blot analysis. Cells in 6-cm dishes were washed with cold PBS and harvested by scraping following addition of lysis buffer (50 mM HEPES, pH 7.4, $250 \mathrm{mM} \mathrm{NaCl}, 1 \%$ Nonidet P-40, $1 \mathrm{mM}$ EDTA, $1 \mathrm{mM} \mathrm{Na} \mathrm{VO}_{4}, 1 \mathrm{mM} \mathrm{NaF}, 1 \mathrm{mM}$ PMSF, $1 \mathrm{mM}$ dithiothreitol and a protease inhibitor cocktail from Roche) for $15 \mathrm{~min}$ on ice. The protein concentration was determined by the bicinchoninic acid assay (BCA Protein Assay kit; Pierce, USA). The samples were boiled for $5 \mathrm{~min}$ and stored at $-20^{\circ} \mathrm{C}$ until use. Equal amounts of protein were electrophoresed on 
Table I. Primers used in the present study.

\begin{tabular}{lll}
\hline Gene & & \multicolumn{1}{c}{ Primer sequence $\left(5^{\prime} \rightarrow 3^{\prime}\right)$} \\
\hline ID1 & $\begin{array}{l}\text { Forward } \\
\text { Reverse }\end{array}$ & $\begin{array}{l}\text { CGTGCTGCTCTACGACATGA } \\
\text { GCTCCAACTGAAGGTCCCTG }\end{array}$ \\
PCNA & Forward & AACCTGCAGAGCATGGACTC \\
& Reverse & TCATTGCCGGCGCATTTTAG \\
Survivin & Forward & TCTCTACATTCAAGAACT \\
& Reverse & TTGAAGCAGAAGAAACAC \\
MMP2 & Forward & TCTTCCCCTTCACTTTCCTG \\
& Reverse & ACTTGCGGTCATCATCGT \\
MMP9 & Forward & GCAGAGATGCGTGGAGAGT \\
& Reverse & CCCTCAAAGGTTTGGAATC \\
CXCR4 & Forward & ATACACTTCAGATAACTAC \\
& Reverse & TAAGAAGATGATGGAGTA \\
$\beta$-actin & Forward & TGGCACCACACCTTCTACA \\
& Reverse & AGCACAGCCTGGATAGCA \\
\hline
\end{tabular}

ID1, DNA-binding protein; CXCR4, CXC chemokine receptor 4.

polyacrylamide gradient gels (10-15\%; Bio-Rad Laboratories) and electro-transferred to membranes. After transfer, the membranes were then blocked in $3 \%$ bovine serum albumin at room temperature for $2 \mathrm{~h}$. The membranes were then incubated overnight at $4^{\circ} \mathrm{C}$ with primary antibodies against PCNA, survivin (Abcam Inc., Cambridge, MA, USA), ID1, MMP2 and MMP9, (Santa Cruz Biotechnology, Santa Cruz, CA, USA), CXCR4 (Cell Signaling Technology, Beverly, MA, USA) and $\beta$-actin (Santa Cruz Biotechnology). The binding of secondary horseradish peroxidase-conjugated antibodies was visualized by enhanced chemiluminescence (ECL Plus, USA).

Immunohistochemical staining. Immunohistochemistry for detection of target protein (ID1, PCNA, survivin, MMP2, MMP9 and CXCR4) expression in the tumor tissues from mice was carried out using the streptavidin-biotin-peroxidase (SP) staining method. The immunostaining SP kit was purchased from Fuzhou Maxim Biotech Company. Paraffinembedded sections were deparaffinized by xylene and dehydrated in graded alcohol. To retrieve antigen, the sections were boiled in $10 \mathrm{mM}$ citrate buffer ( $\mathrm{pH} \mathrm{6.0)}$ for $5 \mathrm{~min}$. The tissue sections were treated with peroxidase blocking agent to block endogenous peroxidase and normal rabbit serum to block non-specific binding sites. The primary antibodies were used at a dilution according to the antibodies manual and were added to adjacent tissue sections and incubated overnight at $4^{\circ} \mathrm{C}$. Secondary antibodies were added to the sections and incubated at room temperature for $10 \mathrm{~min}$. S-P complex was added at room temperature for $10 \mathrm{~min}$, DAB was used for the color reaction, and the sections were counterstained with hematoxylin. Stained sections were viewed and photographed using a fluorescence microscope.

Gelatin zymography. Gelatin zymography was carried out on protein extracts from the HCT116 and SW620 cells. Cells were plated at a density of $1 \times 10^{6}$ in 6 -well plates. After $24 \mathrm{~h}$, the cells were washed with PBS and incubated in $1 \mathrm{ml}$ of serumfree medium for $24 \mathrm{~h}$. The conditioned medium was separated on $10 \% \mathrm{SDS} / \mathrm{PAGE}$ with $1 \mathrm{mg} / \mathrm{ml}$ gelatin incorporated into the gel mixture. Following electrophoresis at $4^{\circ} \mathrm{C}$, the gels were washed 4 times for 15 min each in $2.5 \%$ Triton $\mathrm{X}-100$ to remove the SDS and were incubated for $37 \mathrm{~h}$ at $37^{\circ} \mathrm{C}$ in $50 \mathrm{mM}$ Tris, pH 7.5, $10 \mathrm{mM} \mathrm{CaCl}_{2}, 1 \mu \mathrm{M} \mathrm{ZnCl}_{2}$ and $150 \mathrm{mM}$ $\mathrm{NaCl}$. Afterwards, the gels were fixed and stained with $0.5 \%$ Coomassie blue in 30\% isopropanol/10\% acetic acid for $1 \mathrm{~h}$, then destained in 30\% isopropanol $/ 10 \%$ acetic acid. The stain was washed out with water until clear bands were observed.

In vivo nude mouse study. Male nude mice (BALB/c nu/nu), 4-6 weeks old, weighing 16-19 g, were purchased from the Shanghai SLAC Laboratory Animal Co., Ltd. (Shanghai, China). All experiments were approved by the Animal Ethics Committee of Fujian Medical University. To establish CRC xenografts, six mice received $3 \times 10^{6}$ control and shID1-KD HCT116 cells in a final volume of $0.1 \mathrm{ml}$ PBS by subcutaneous injection in the right and left groin, respectively. Measurement of the resulting tumor xenografts began when the size was $>2 \mathrm{~mm}$ in diameter and was carried out thereafter every three days. Tumor volumes were calculated using the following formula: Volume $=\left[\right.$ length $(\mathrm{mm}) \times$ width $\left.^{2}\left(\mathrm{~mm}^{2}\right)\right] / 2$. Mice were sacrificed 21 days after the injections and immediately weighed.

For the experimental liver metastasis assays, cells were injected into the spleen $\left(5 \times 10^{6}\right.$ cells/mouse). The mice were placed in the right lateral decubitus position, an incision in the abdominal wall on the left side was made, the spleen was exteriorized, and the cells were injected into the spleen. Mice were sacrificed at day 28. Tumor samples from the site of the tumor injection and from livers (metastasis target organ) were shock-frozen in liquid nitrogen, formalin-fixed and paraffinembedded.

Statistical analysis. The data represent means \pm SD from at least six independent experiments. Statistical analysis was performed with the Student's t-test at a significance level of $\mathrm{P}<0.05$. All data analyses were conducted by use of the SPSS 20.0 statistical software package.

\section{Results}

Downregulation of IDI decreases proliferation and induces apoptosis. In order to determine the function of ID1 in CRC, we established stable cancer cell lines with ID1 knockdown. To this end, 4 independent lentiviral shRNAs targeting the ID1 gene were evaluated for their knockdown efficiency. Quantitative PCR (qPCR) analysis demonstrated that ID1 transcript in the HCT116 cell line was affected by the different ID1 shRNAs to varying degrees of reduction $(50-85 \%$, data not shown). We used the lentivirus containing the most efficient shRNA to infect the HCT116 and SW620 cells and found a robust depletion of ID1 mRNA in both cell lines (Fig. 1A). Subsequently, we generated stable pools of HCT116 and SW620 cells with shID1 and control shRNA. Western blot analysis demonstrated an $\sim 60-70 \%$ reduction in ID1 protein in the ID1-KD cells as compared to the control cells (Fig. 1B). 

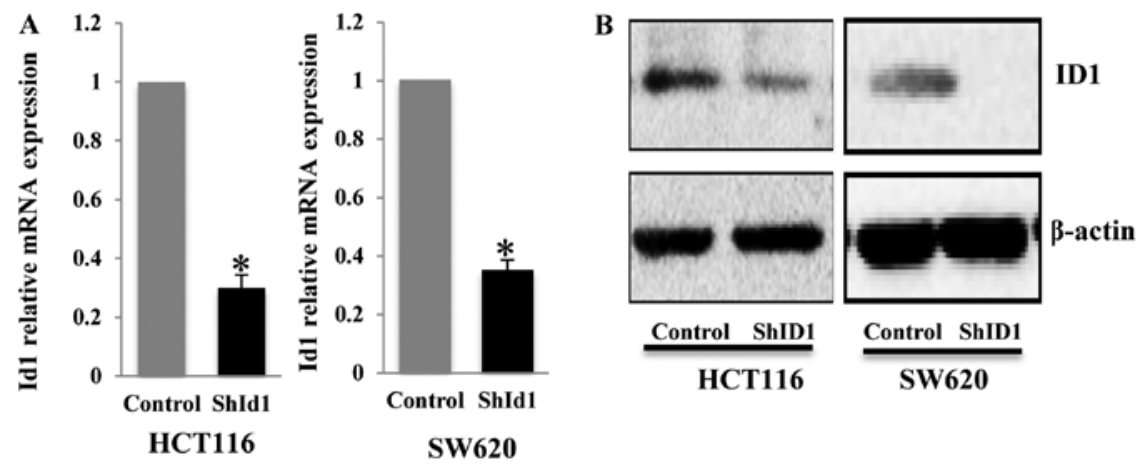

Figure 1. Knockdown of ID1 in colon cancer cells. (A and B) ID1-RNAi transfection silenced the mRNA and protein expression of ID1 in both the HCT116 and SW620 cell lines. ID1 mRNA and protein levels were assessed by (A) real-time RT-PCR and (B) western blotting.

A
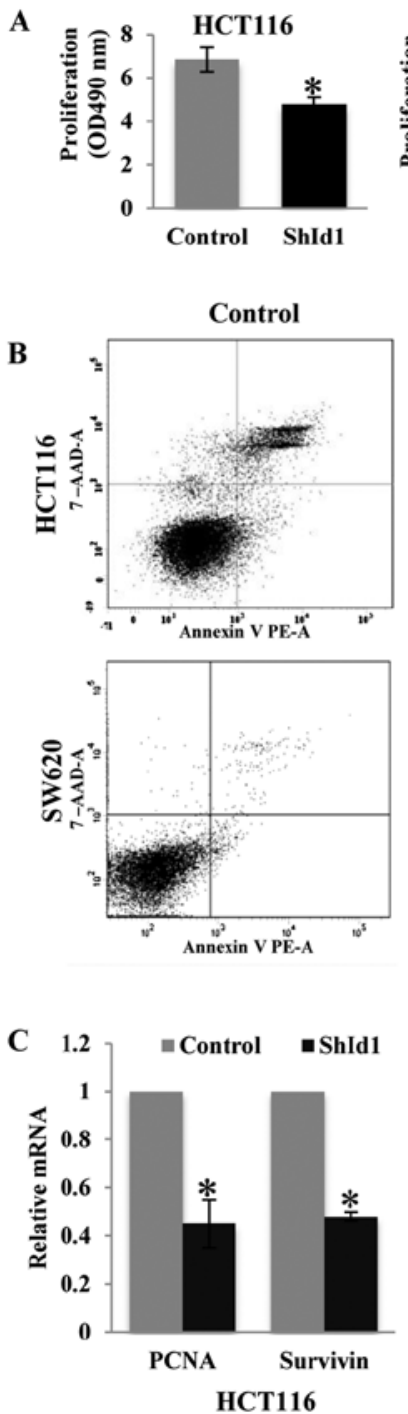
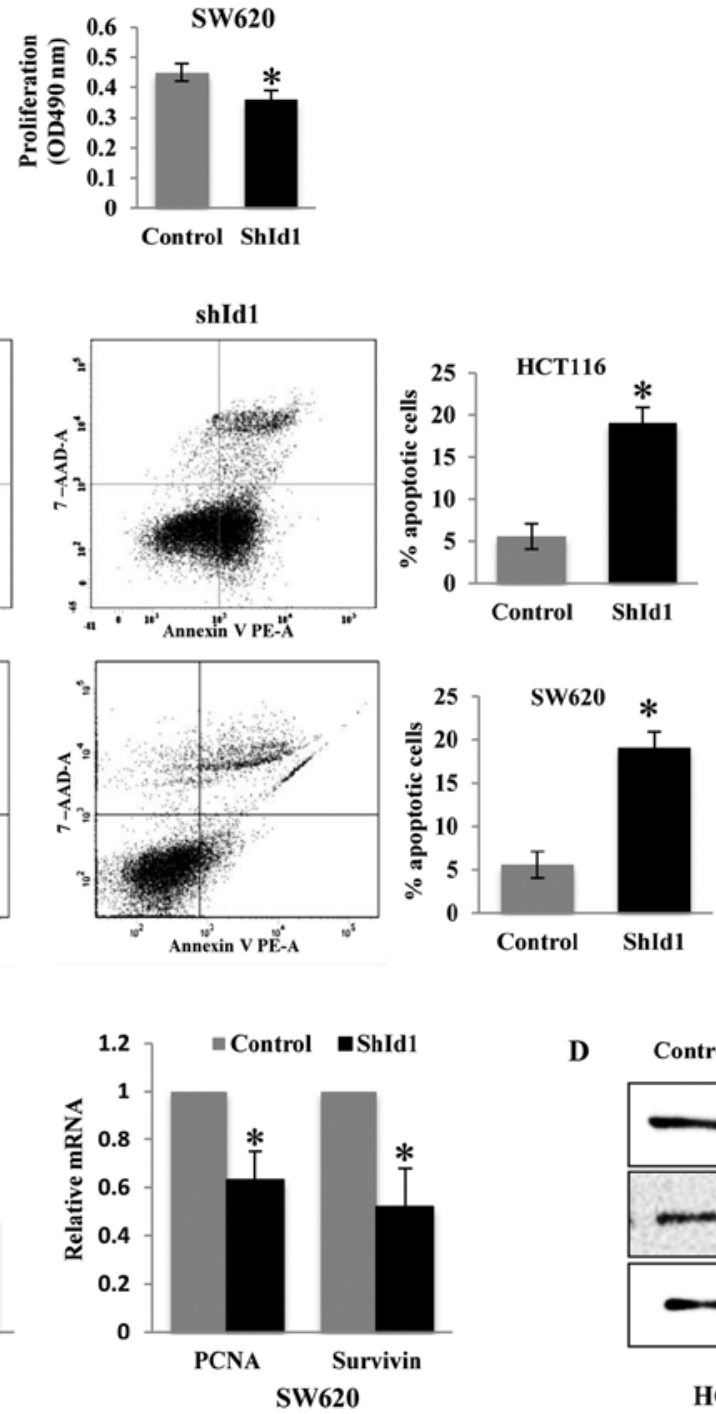

D

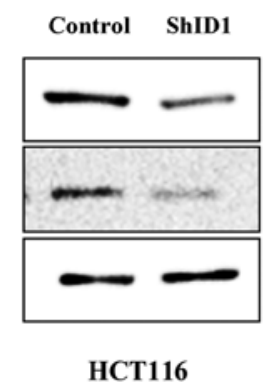

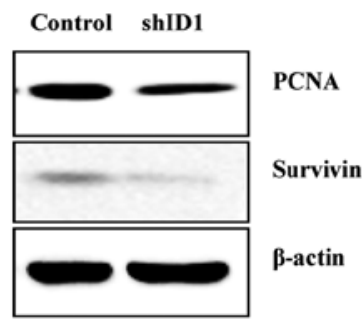

SW620

Figure 2. Downregulation of ID1 reduces cell proliferation and induces apoptosis of CRC cells. (A) MTS proliferation assay showing significantly decreased cell proliferation by ID1 knockdown, when compared with the average of controls at $48 \mathrm{~h}$ after plating. (B) Flow cytometric assays were performed to detect changes in apoptosis after stable knockdown of ID1 and in the controls. ID1-KD HCT116 and SW620 cells exhibited higher apoptosis rates when compared with the rates in the control cells. ID1 knockdown decreased (C) PCNA and survivin mRNA and (D) protein expression as assessed by q-PCR and western blotting, respectively, when compared with these levels in the control cells. The results are expressed as the means \pm SE. "P $<0.05$ vs. control. KD, knockdown.

To assess the function of ID1 in the proliferation of CRC cells, we performed an MTS assay to determine the cell proliferation rate in the ID1-KD and control cells. As shown in Fig. 2A, ID1 knockdown in both HCT116 and SW620 cells resulted in a significant decrease in cell proliferation when compared to the controls, suggesting its pivotal role in cell 
A

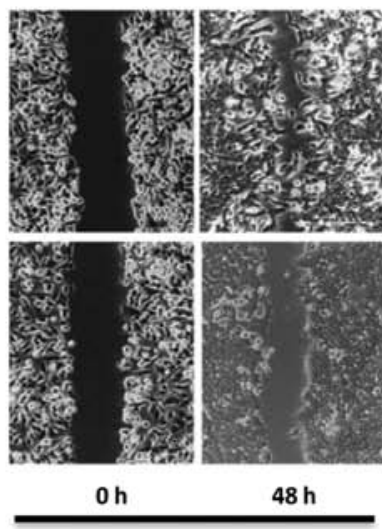

HCT116

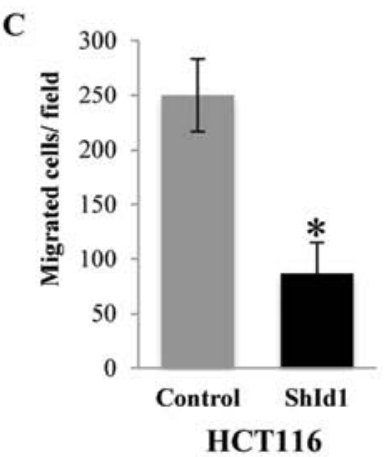

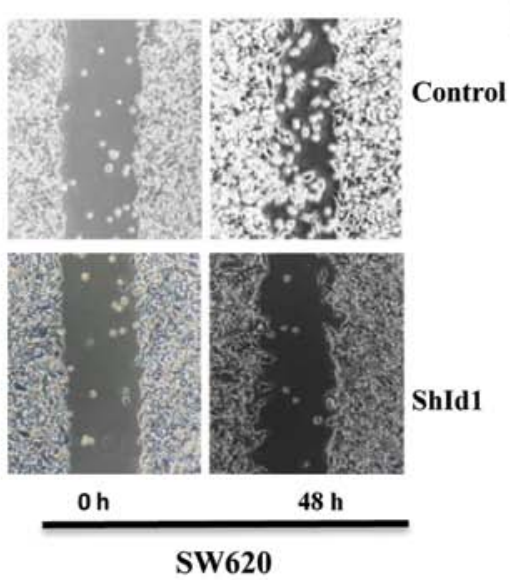

SW620

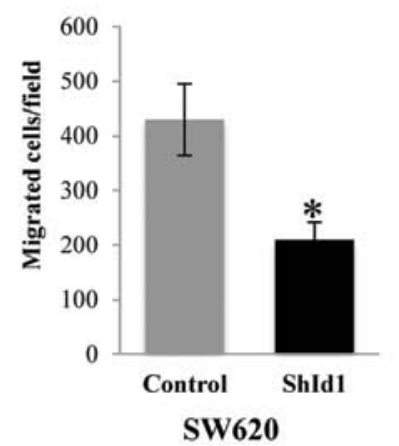

B

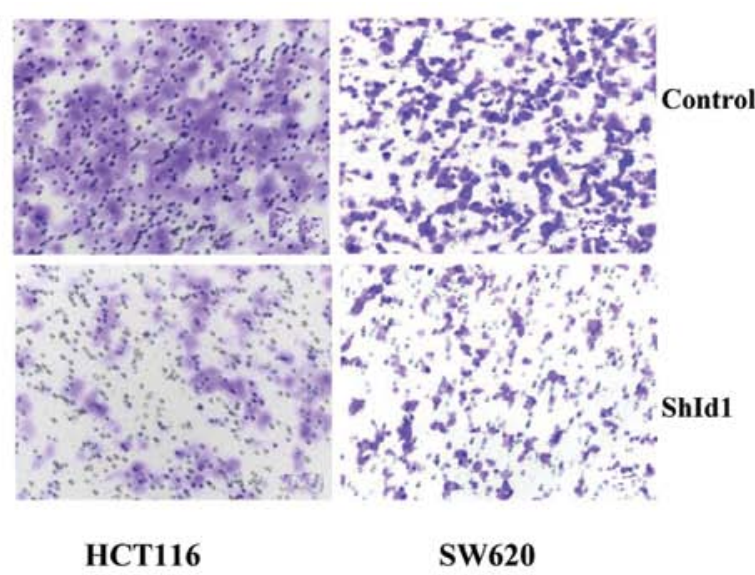

D

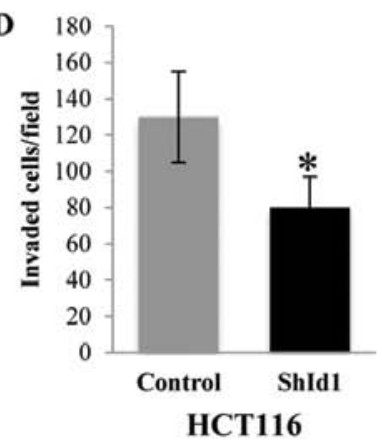

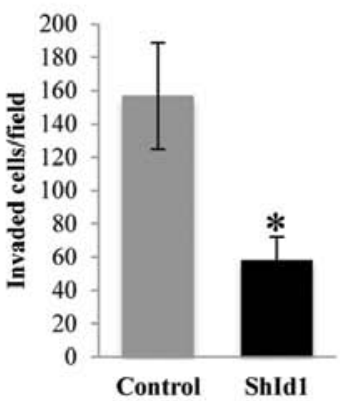

SW620

Figure 3. Downregulation of ID1 expression inhibited the migration/invasion ability of colon cancer cells. (A) Light microscopy images are shown immediately after scratching of the monolayer $(0 \mathrm{~h})$ and after $48 \mathrm{~h}$. Cells moved more slowly over the wound after ID1 knockdown. The cells that (B and C) migrated through the inserts or (D) invaded through the Matrigel-coated collagen inserts were counted under a light microscope at x100 magnification. ID1 knockdown inhibited both the migration and invasion capacity of the HCT116 and SW620 cells.

growth. We then aimed to ascertain whether depletion of ID1 induces apoptosis in CRC cells. To detect early and late apoptosis rates in shID1 knockdown CRC cells, the Annexin V-PE and 7-AAD double staining assay was used. The early (Annexin $\mathrm{V}^{+} / 7 \mathrm{AAD}^{-}$) and late apoptosis (Annexin $\mathrm{V}^{+} / 7-\mathrm{AAD}^{+}$) rates were significantly increased in the ID1-KD cells when compared to these rates in the controls (Fig. 2B). Two genes associated with proliferation (PCNA) and survival (survivin) were examined by real-time PCR and western blotting. Consistent with these observations, the mRNA quantities of PCNA and survivin were significantly decreased in the ID1-KD HCT116 and SW620 cells ( $<<0.05)$ (Fig. 2C). Western blotting also confirmed that protein levels of PCNA and survivin were reduced in the ID1-KD cells when compared to the levels in the control cells (Fig. 2D). Collectively, these data indicate that ID1 plays an important role in CRC cell proliferation and apoptosis and downregulation of ID1 forms a limiting factor for growth of these cancer cells.

Downregulation of IDI reduces motility, migration and invasion capacity of CRC cells. To analyze a possible effect of ID1-KD on the migration of CRC cells, cell migration ability was assessed with a scratch assay. As shown in Fig. 3A, ID1-KD cells migrated through the wound scratch more slowly than the control cells. Migration/invasion capacity was tested by Transwell/invasion assays. We also found a significant decrease in cellular migration/invasion capacity in cells with
ID1 knockdown. The decrease in the migration/invasion of ID1-KD cells was statistically significant ( $\mathrm{P}<0.05$ vs. control group) (Fig. 3B-D).

MMP2 and MMP9 belong to the gelatinase subfamily of matrix metalloproteinases, which are known to be involved in invasion of cancer cells. We then tested the expression of these markers in the control and ID1-KD cells. Both qPCR and western blot assays revealed that mRNA and protein of MMP2 and MMP9 were significantly decreased in the ID1-KD HCT116 and SW620 cells (Fig. 4A and B). Consistently, gelatin zymography revealed an anticipated decrease in MMP2 and MMP9 activity in the ID1-KD cells compared with the control cells (Fig. 4C). In addition, we examined the alteration in CXCR4 in the ID1-KD cells. CXCR4 is a chemokine receptor closely linked to cancer cell growth, migration and invasion in CRC and other cancer types (23). We found that CXCR4 mRNA and protein levels were concurrently decreased in the HCT116 and SW620 cells with ID1 knockdown (Fig. 4A and B). These results revealed a possible molecular mechanism underlying ID1-mediated cell migration and invasion.

CXCR4 reverses the negative effect of the downregulation of IDI in regards to migration and invasion of CRC cells. Our data suggest that the attenuation of motility, migration and invasion capacity of CRC cells by ID1 depletion are attributable to the compromised function of MMP proteins and CXCR4. To substantiate this hypothesis, CXCR4, as a representative, 

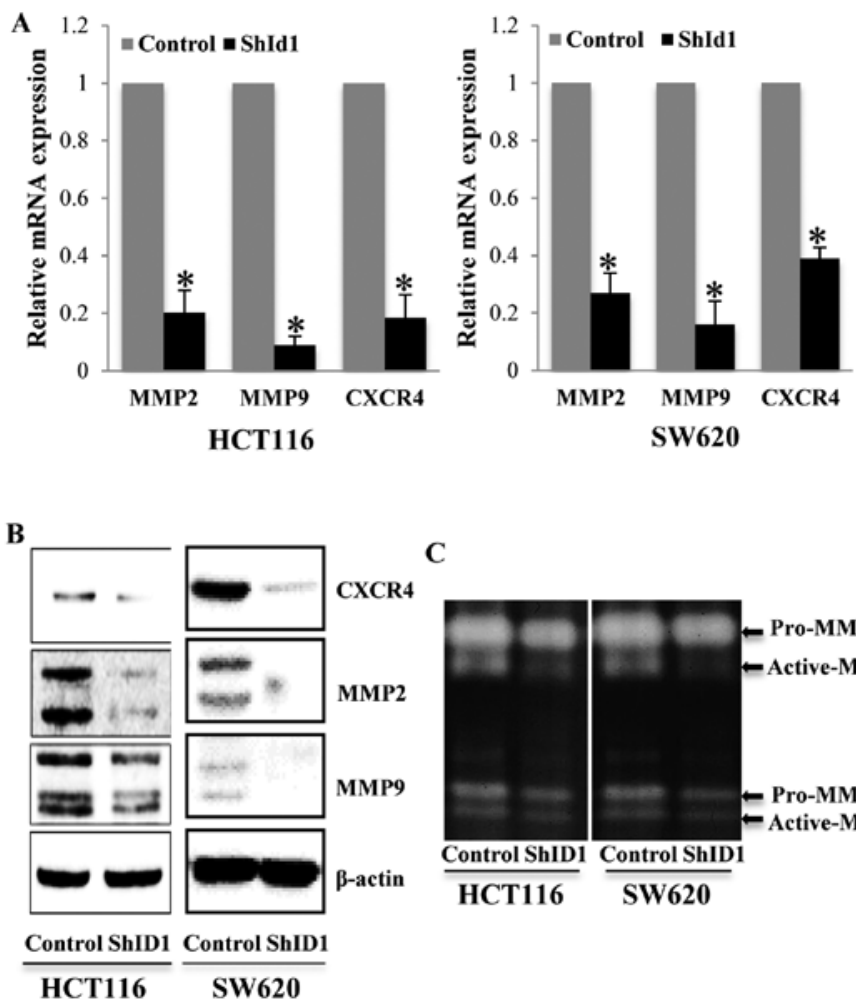

C

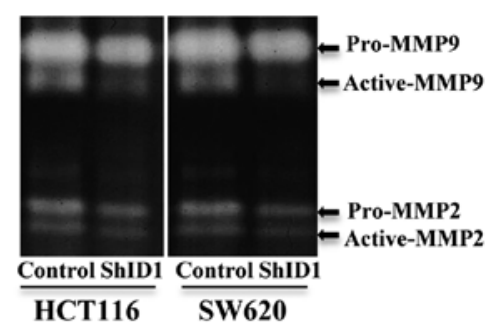

Figure 4. ID1 knockdown reduces expression of CXCR4, MMP2 and MMP9 in colon cancer cells. (A) Levels of CXCR4, MMP2 and MMP9 mRNA were significantly decreased in the ID1-KD cells compared to the controls. (B) Protein levels of CXCR4, MMP2 and MMP9 were reduced in the ID1-KD cells as compared to controls in the western blot assay. (C) Gelatin zymography for determination of MMP2 and MMP9 activity. The results are expressed as the means \pm SE. ${ }^{*} \mathrm{P}<0.05$ vs. control. KD, knockdown; CXCR4, CXC chemokine receptor 4.

A
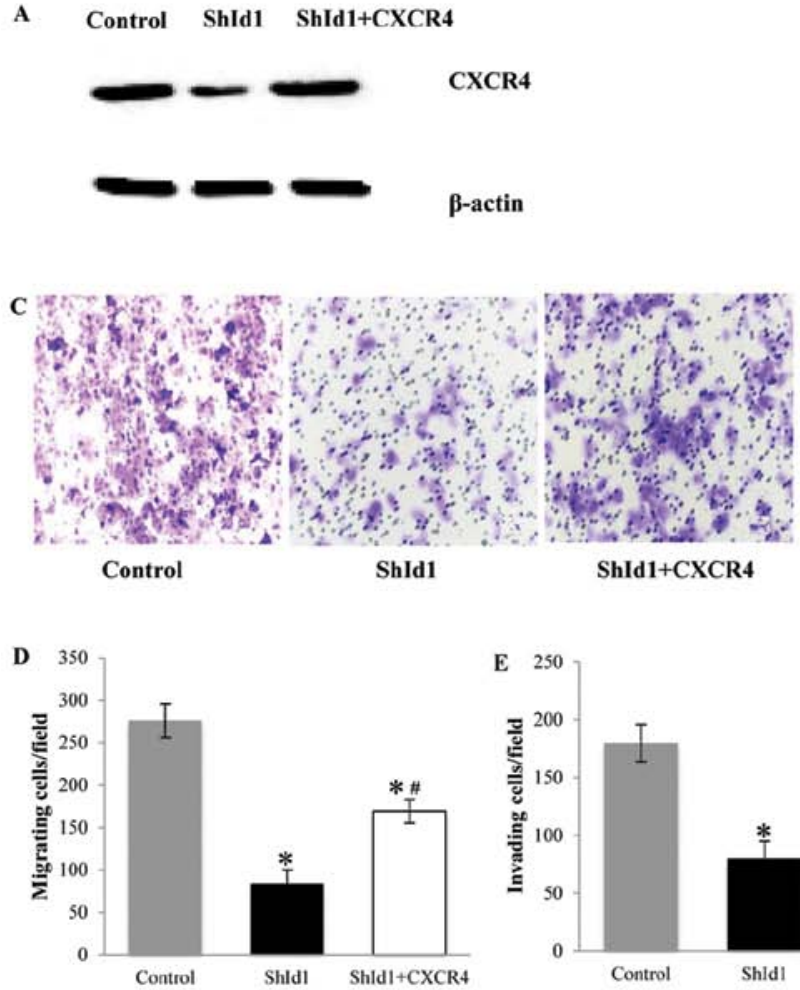

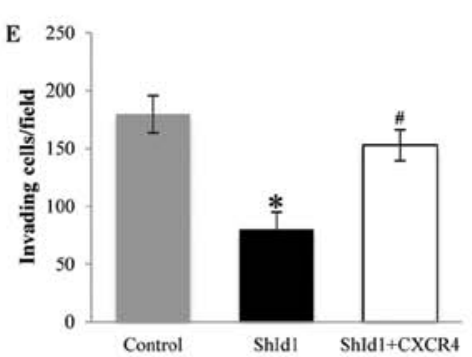

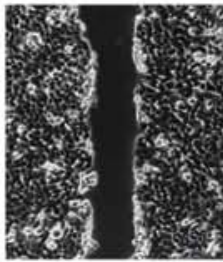
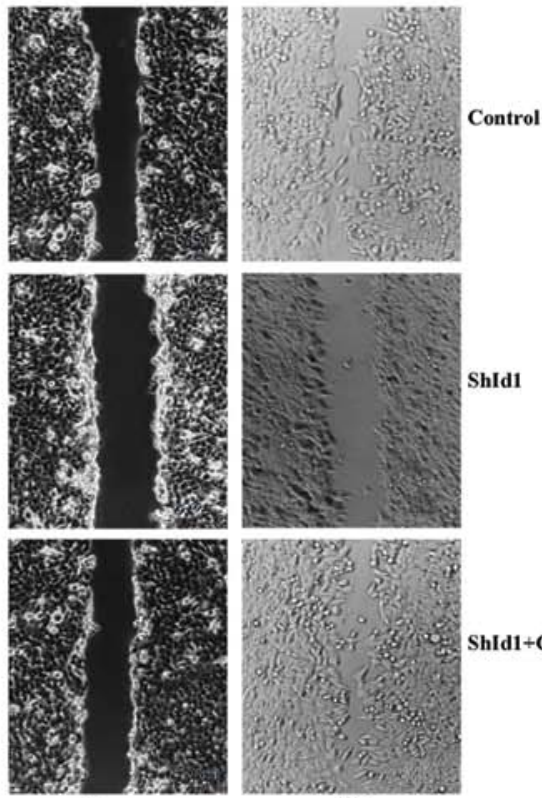

$0 \mathrm{~h}$

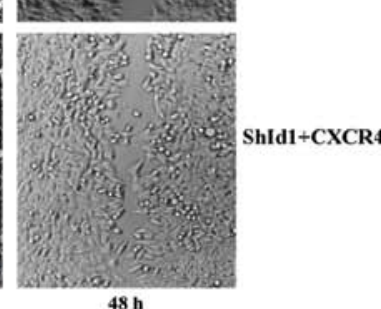

Figure 5. ID1 knockdown decreases the migration and invasion capacity of HCT116 cells partly through CXCR4 downregulation. (A) Western blot analysis of CXCR4 protein levels in controls and ID1-KD/CXCR4-overexpressing cells (shId1+CXCR4) is shown. (B) Light microscopy images are shown immediately after scratching of the monolayer at $0 \mathrm{~h}$ and after $48 \mathrm{~h}$. In both cell lines expression of CXCR4 caused cells to move faster than the ID-KD cells. (C-E) The cells that migrated or invaded through the inserts were counted under a light microscope at x100 magnification. Overexpression of CXCR4 partially rescued the effect of ID1 knockdown on (C and D) migration and (E) invasion capacity. The results are expressed as the means \pm SE. ${ }^{*} \mathrm{P}<0.05$ vs. control, ${ }^{*} \mathrm{P}<0.05$ vs. ID1-KD cells. CXCR4, CXC chemokine receptor 4; KD, knockdown. 


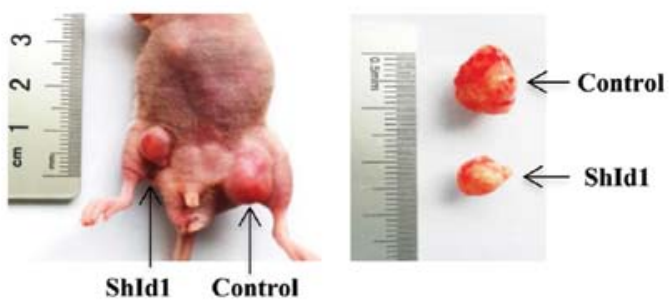

C

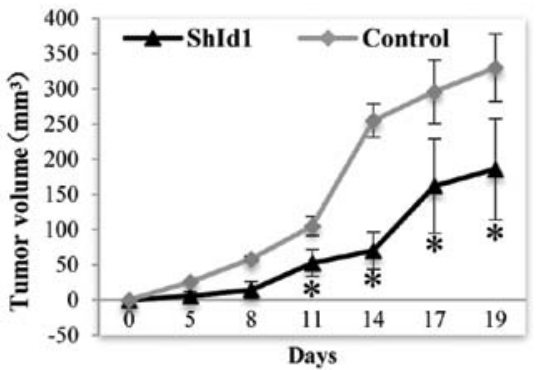

E

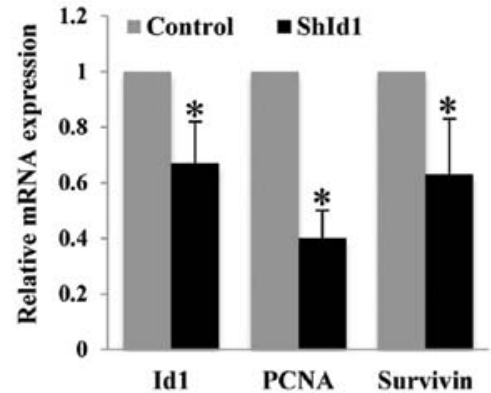

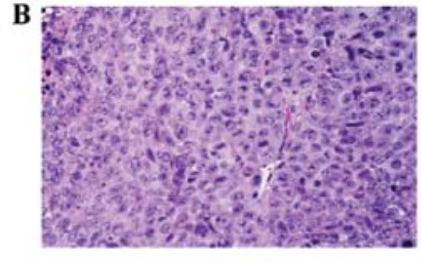

H\&E

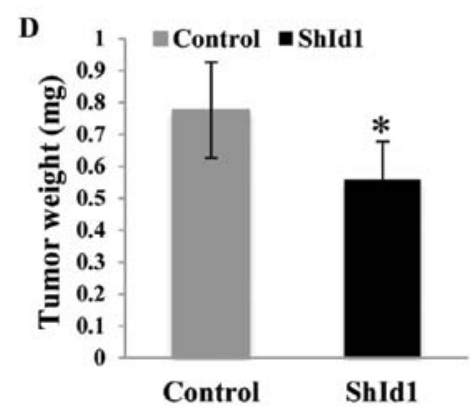

$\mathbf{F}$

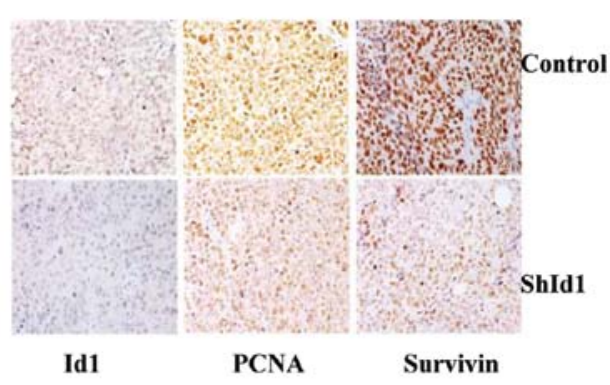

Figure 6. ID1 knockdown inhibits tumor growth in vivo. (A and B) Images of representative tumors from mice injected with control or HCT116 cells with stable ID1-knockdown. (A) Cells with ID1 KD produced smaller tumor masses than did the control cells. (C) The tumor growth of ID1-KD cells was slower compared with the control cells. The difference was statistically significant at day 11. (D) Mean tumor weights for ID1-KD cells and controls are presented. The tumor weight of the ID1-KD tumors was significantly less than the control cell tumors. (E) ID1, PCNA and survivin mRNA expression was evaluated by q-PCR. These mRNAs were all significantly reduced in subcutaneous tumor samples of the ID1-KD tumors compared with the controls. (F) Immunohistochemical staining of ID1, PCNA and survivin expression in the harvested subcutaneous tumor samples (x400 magnifications). ID1-KD tumors had lower levels of these proteins compared with the controls. The results are expressed as the means $\pm \mathrm{SE}$. ${ }^{*} \mathrm{P}<0.05$ vs. control. KD, knockdown.

was further investigated by using a genetic rescue experiment, in which CXCR4 was compensated by its exogenous protein in ID1-KD HCT116 cells. As shown in Fig. 5A, CXCR4 in the ID1-KD cells was restored to a similar level as that in the controls by transfection of a CXCR4-overexpression vector. Consequently, ID1-KD/CXCR4-overexpressing (OE) cells showed higher motility in the scratch assay than the ID1-KD counterparts (Fig. 5B). Moreover, in the Transwell culture assays, CXCR4 restoration significantly improved migration (Fig. 5C and D) and invasion of ID1-KD cells (Fig. 5E). Although CXCR4 rescue did not fully reverse the effect of ID1 knockdown, this partial rescue function in regards to the migration and invasion capacity indicated that CXCR4 indeed played an important role in the altered tumor cell motility by ID1 knockdown. This finding also suggests that other factors are likely to orchestrate this process.

Downregulation of ID1 inhibits tumor growth of HCT116 cells in a xenograft mouse model in vivo. The effects of ID1 knockdown on tumor growth in vivo were first investigated in a subcutaneous tumor model using HCT116 cells. In agreement with the in vitro findings, ID1-KD cells formed smaller tumors than the control cells (Fig. 6A-D). The effect of shId1 on the silencing of the Id1 gene in the xenografted tumors was evaluated by real-time PCR and immunohistochemical analysis. As shown in Fig. 6E and F, a >50\% ID1 mRNA and protein reduction was found in the ID1-KD tumors compared with the controls $(\mathrm{P}<0.05)$. In addition, PCNA and survivin mRNA levels were significantly decreased in the xenografted tumors $(\mathrm{P}<0.05)$. The protein levels of PCNA and survivin assayed by immunohistochemistry were also decreased in the ID1-KD tumor sections (Fig. 6F).

Downregulation of ID1 inhibits metastasis of HCT116 cells in a liver metastasis mouse model. Next, we evaluated the effect of ID1 on the in vivo liver metastasis of HCT116 KD tumors. In line with our in vitro findings, both qPCR and immunohistochemistry showed decreased MMP2, MMP9 
A

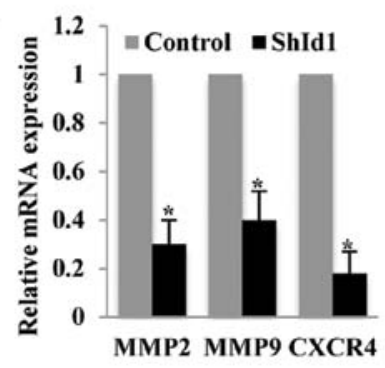

C

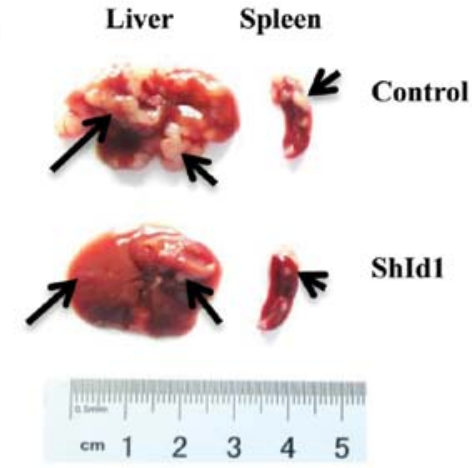

D

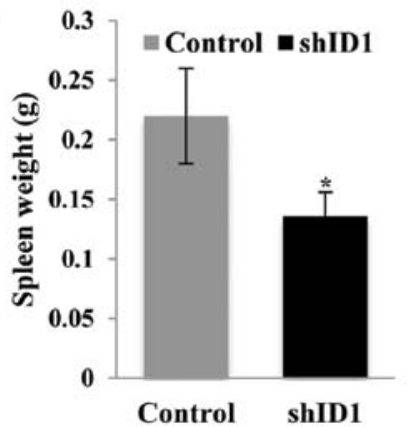

B
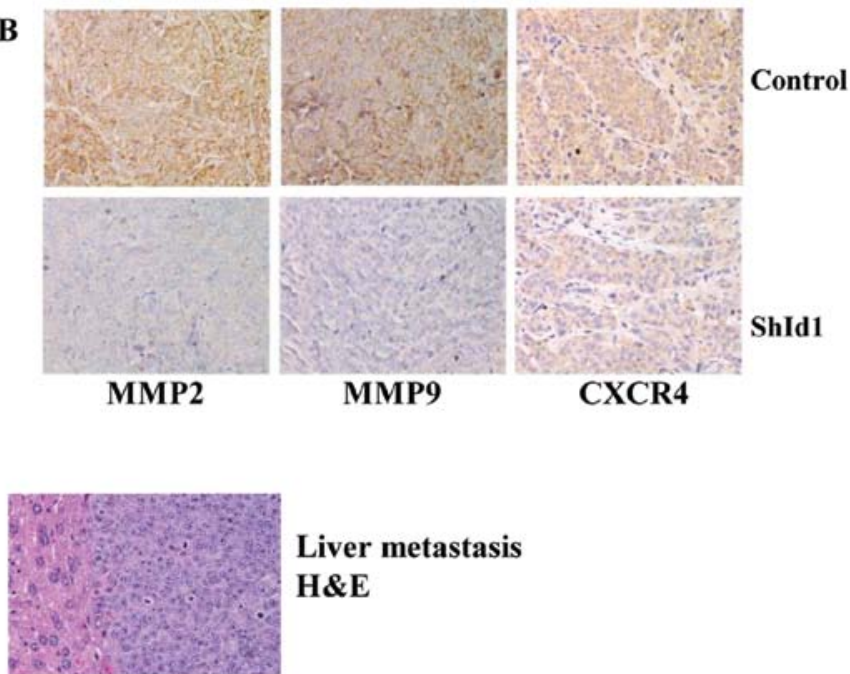

Liver metastasis

H\&E

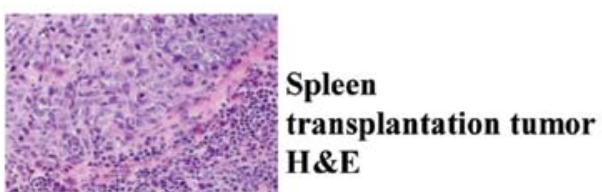

E

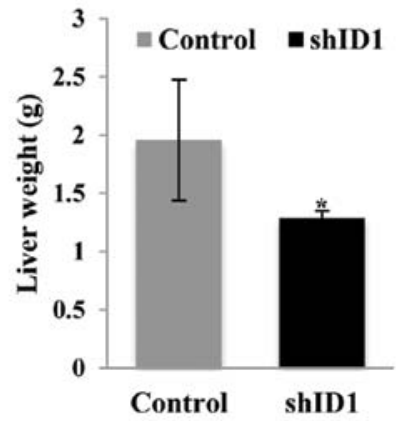

Figure 7. ID1 knockdown reduces liver metastasis in vivo. (A) MMP2, MMP9 and CXCR4 mRNA expression levels were significantly reduced in the subcutaneous tumor samples of ID1-KD cells compared with the controls. (B) Immunohistochemical staining of MMP2, MMP9 and CXCR4 expression in harvested subcutaneous tumor samples (x400 magnification). (C) Intrasplenic implantation of ID1-KD HCT116 cells or control cells. The tumors in the spleens are shown. ID1 knockdown decreased the formation of liver metastases, as exemplified for one mouse per group. (D) Mean liver and spleen weights are shown. Both the liver and spleen weights were significantly reduced in the ID1-KD group compared to the control group. The results are expressed as the means \pm SE. ${ }^{*} \mathrm{P}<0.05$ vs. control. KD, knockdown; CXCR4, CXC chemokine receptor 4.

and CXCR4 mRNA and protein in the ID1-KD subcutaneous tumors compared to the control group (Fig. 7A and B). We implanted HCT116 cells into the spleen which led to tumor growth in the spleen and metastasis to the liver. Twenty-eight days after tumor cell injection, all animals in the control group developed liver metastases (10/10). In contrast, only 6/10 animals in the ID1-KD group developed liver metastases that were barely visible $(\mathrm{P}<0.05)$. As compared to the vector control, ID1 profoundly suppressed liver metastases in the mice (Fig. 7C and D).

\section{Discussion}

The principal finding in the present study was that knockdown of ID1 protein expression correlates with the growth arrest of CRC cell lines and the suppression of hepatic metastasis of
CRC tumors in a mouse model. The study also emphasized the role of ID1/CXCR4 as a positively regulatory axis required for CRC cell proliferation and migration and tumor invasion. This finding is consistent with these of recent reports on the inhibitive effect of ID1 and ID3 gene downregulation on CRC hematogenous metastasis at the early stage of the tumor (21). The results imply that the ID1 gene could be a therapeutic target for CRC. Our present study indicated that ID1 knockdown caused reduced proliferation and induced apoptosis of CRC cells in vitro and restrained tumor growth in vivo. This can be interpreted along with recent studies of the tumorigenic effect of ID1 in keratinocytes (22) and in a variety of human tumors $(23,24)$, which suggest that an elevated ID1 protein level may promote cell proliferation, inhibit cellular apoptosis, and repress differentiation, thereby leading to the onset and progression of CRC. 
Since survivin was reported to be a downstream regulator in the ID1/PI3K/Akt/NF- $\mathrm{B} /$ survivin signaling pathway for endothelial progenitor cell proliferation, and CRC tumor growth and malignant metastasis $(25,26)$, the fact that ID1 knockdown induced a decrease in survivin expression, may indicate that activation of survivin is a consequence of the expression of ID1 in CRC cell lines. It has been suggested that PCNA is a proliferation marker of tumors and is critical for DNA synthesis (27). We showed that ID1 knockdown decreased PCNA expression at both the mRNA and protein levels. However, no significant change in the cell cycle was noted following ID1 knockdown (data not shown). Thus, there may be alternative mechanisms that remain to be identified in the stabilization of DNA synthesis. To some extent, these results, together with previous studies, imply that modulation of ID1 expression positively interferes with the functions of PCNA and survivin and subsequently inhibits tumor growth and invasion in CRC.

Many reports have addressed the role of ID1 in tumor metastasis. For example, ID1 was found to elevate the expression of epithelial mesenchymal markers in nicotine and EGF-induced proliferation, migration and invasion of nonsmall cell lung cancer (23). Similarly, ID1 was found to be positively associated with the migratory and invasive features of breast cancer cells by enhancing epithelial-mesenchymallike markers (28). A clinical observation indicated that ID1 expression was strongly associated with lymph node metastasis in patients with CRC (13). Our results support recent study by O'Brien et al (20) who demonstrated a significant reduction in tumor growth and hepatic metastatic burden following double-gene ID1 and ID3 knockdown in CRC cell lines in a mouse model. We also found that ID1 knockdown suppressed the expression and activity of the matrix metalloproteinases, MMP2 and MMP9, in vitro, suggesting that ID1-KD may protect against angiogenic destruction of the extracellular matrix of regional blood vessels during adjacent and distal invasion and metastasis (29-31). ID1 knockdown induced suppression of angiogenesis may likely be explained in part by the ID1/NF- $\mathrm{B} / \mathrm{MMP}-2$ or ID1/PI3K/Akt signaling pathways identified in ovarian cancer (32) and integrins $(\alpha 3$, $\alpha 6$ and $\beta 1$ )/laminin adhesion in pancreatic cancer (33).

Another finding of the present study indicated that gross overexpression of CXCR4 reversed the ID1 knockdownsuppressive effects on cell proliferation and metastasis. Metastasis-related molecular events have been explored in several studies (34-36) of which one of the chemokine family members, CXCR4, was significantly higher in liver metastasis compared with primary CRC tumor tissue and was found to correlate with reduced overall median patient survival after liver metastasis (34). In addition, a specific ligand of CXCR4, stromal cell-derived factor-1 (SDF-1), was found to highly express in tumor-adherent stromal cells of metastatic organs such as the liver, lung and lymph nodes (36). Notably, we demonstrated both in vitro and in vivo, significant reductions in CXCR4 expression and hepatic metastasis burden of CRC cells following inactivation of ID1. The fact that overexpression of CXCR4 largely restored the migration and invasion capacities of the HCT116 ID1 knockdown cells, probably represents a positive loop of feedback underlying the interaction of ID1 and CXCR4 in CRC migration and invasion ability.
Taken together, the present data suggest that highly enforced expression of ID1 causes CRC cell growth arrest and retards metastatic progression in a CXCR4-dependent manner.

In summary, our studies in vitro and in vivo confirmed the regulatory role of ID1 in the proliferation and metastasis of CRC. The findings provide initial evidence that downregulation of ID1 reduces CRC migration and invasion partly through reduced CXCR4 expression in tumor cells, suggesting the rationale of the ID1 protein as a clinical target of CRC treatment. In order to elucidate its oncogenic function, additional studies are needed to explore the network of ID1 within CXCR4/CD133-related stromal self-renewal in CRC malignancy (37).

\section{Acknowledgements}

This study was supported by a grant from the National Natural Science Foundation of China (grant no. 81250002). We would like to thank Professor Xin Lin for his expert suggestions in experiments.

\section{References}

1. Siegel R, Naishadham D and Jemal A: Cancer statistics, 2013. CA Cancer J Clin 63: 11-30, 2013.

2. Christofori, G: New signals from the invasive front. Nature 441: 444-450, 2006

3. Benezra R, Davis RL, Lockshon D, et al: The protein Id: a negative regulator of helix-loop-helix DNA binding proteins. Cell 61: 49-59, 1990.

4. Prabhu S, Ignatova A, Park ST and Sun XH: Regulation of the expression of cyclin-dependent kinase inhibitor p21 by E2A and Id proteins. Mol Cell Biol 17: 5888-5896, 1997.

5. Iavarone A, Garg P, Lasorella A, et al: The helix-loop-helix protein Id-2 enhances cell proliferation and binds to the retinoblastoma protein. Genes Dev 8: 1270-1284, 1994.

6. Lasorella A, Iavarone A and Israel MA: Id2 specifically alters regulation of the cell cycle by tumor suppressor proteins. Mol Cell Biol 16: 2570-2578, 1996.

7. Lasorella A, Uo T and Iavarone A: Id proteins at the cross-road of development and cancer. Oncogene 20: 8326-8333, 2001.

8 . Yokota Y and Mori S: Role of Id family proteins in growth control. J Cell Physiol 190: 21-28, 2002.

9. Lyden D, Young AZ, Zagzag D, et al: Id1 and Id3 are required for neurogenesis, angiogenesis and vascularization of tumour xenografts. Nature 401: 670-677, 1999.

10. Norton JD: ID helix-loop-helix proteins in cell growth, differentiation and tumorigenesis. J Cell Sci 113: 3897-3905, 2000.

11. Sikder HA, Devlin MK, Dunlap S, et al: Id proteins in cell growth and tumorigenesis. Cancer Cell 3: 525-530, 2003.

12. Yang HY, Liu HL, Liu GY, et al: Expression and prognostic values of Id-1 and Id-3 in gastric adenocarcinoma. J Surg Res 167: 258-266, 2011.

13. Zhao ZR, Zhang ZY, Zhang H, et al: Overexpression of Id-1 protein is a marker in colorectal cancer progression. Oncol Rep 19: 419-424, 2008.

14. Forootan SS, Wong YC, Dodson A, et al: Increased Id-1 expression is significantly associated with poor survival of patients with prostate cancer. Hum Pathol 38: 1321-1329, 2007.

15. Luo KJ, Wen J, Xie X, et al: Prognostic relevance of Id-1 expression in patients with resectable esophageal squamous cell carcinoma. Ann Thorac Surg 93: 1682-1688, 2012.

16. Sun W, Guo MM, Han P, et al: Id-1 and the $\mathrm{p} 65$ subunit of NF- $\kappa \mathrm{B}$ promote migration of nasopharyngeal carcinoma cells and are correlated with poor prognosis. Carcinogenesis 33: 810-817, 2012.

17. Cheng YJ, Tsai JW, Hsieh KC, et al: Id1 promotes lung cancer cell proliferation and tumor growth through Akt-related pathway. Cancer Lett 307: 191-199, 2011.

18. Sumida T, Murase R, Onishi-Ishikawa A, et al: Targeting Id1 reduces proliferation and invasion in aggressive human salivary gland cancer cells. BMC Cancer 13: 141, 2013. 
19. Wilson JW, Deed RW, Inoue T, et al: Expression of Id helix-loop helix proteins in colorectal adenocarcinoma correlates with p53 expression and mitotic index. Cancer Res 61: 8803-8810, 2001

20. O'Brien CA, Kreso A, Ryan P, et al: ID1 and ID3 regulate the self-renewal capacity of human colon cancer-initiating cells through p21. Cancer Cell 21: 777-792, 2012.

21. Okaji Y, Tsuno NH, Kitayama J, et al: Effects of down-regulating the Id genes in human colorectal cancer cells on early steps of haematogenous metastasis. Eur J Cancer 42: 668-673, 2006.

22. Alani RM, Young AZ and Shifflett CB: Id1 regulation of cellular senescence through transcriptional repression of p16/Ink4a. Proc Natl Acad Sci USA 98: 7812-7816, 2001.

23. Pillai S, Rizwani W, Li X, et al: ID1 facilitates the growth and metastasis of non-small cell lung cancer in response to nicotinic acetylcholine receptor and epidermal growth factor receptor signaling. Mol Cell Biol 31: 3052-3067, 2011.

24. Li W, Zhang CH, Hong YL, et al: Inhibitor of DNA-binding-1/ inhibitor of differentiation-1 (ID-1) is implicated in various aspects of gastric cancer cell biology. Mol Biol Rep 39: 3009-3015, 2012.

25. Li W, Wang H, Kuang CY, et al: An essential role for the Id1/ $\mathrm{PI} 3 \mathrm{~K} / \mathrm{Akt} / \mathrm{NF} \mathrm{B} /$ survivin signalling pathway in promoting the proliferation of endothelial progenitor cells in vitro. Mol Cell Biochem 363: 135-145, 2012.

26. Ye Q, Cai W, Zheng Y, et al: ERK and AKT signaling cooperate to translationally regulate survivin expression for metastatic progression of colorectal cancer. Oncogene: Apr 29, 2013 (Epub ahead of print). doi: 10.1038/onc.2013.122.

27. Zhong W, Peng J, He H, et al: Ki-67 and PCNA expression in prostate cancer and benign prostatic hyperplasia. Clin Invest Med 31: E8-E15, 2008.

28. Tobin NP, Sims AH, Lundgren KL, Lehn S and Landberg G: Cyclin D1, Id1 and EMT in breast cancer. BMC Cancer 11: 417 2011.
29. Li B, Tsao SW, Li YY, et al: Id-1 promotes tumorigenicity and metastasis of human esophageal cancer cells through activation of PI3K/AKT signaling pathway. Int J Cancer 125: 2576-2585, 2009.

30. Ling YX, Tao J, Fang SF, Hui Z, and Fang QR: Downregulation of Id1 by small interfering RNA in prostate cancer PC 3 cells in vivo and in vitro. Eur J Cancer Prev 20: 9-17, 2011.

31. Sato Y: Molecular diagnosis of tumor angiogenesis and antiangiogenic cancer therapy. Int J Clin Oncol 8: 200-206, 2003

32. Su Y, Gao L, Teng L, Wang Y, et al: Id1 enhances human ovarian cancer endothelial progenitor cell angiogenesis via PI3K/Akt and NF- $\kappa \mathrm{B} / \mathrm{MMP}-2$ signaling pathways. J Transl Med 11: 132, 2013.

33. Shuno Y, Tsuno NH, Okaji Y, et al: Id1/Id3 knockdown inhibits metastatic potential of pancreatic cancer. J Surg Res 161: 76-82, 2010.

34. Kim J, Takeuchi H, Lam ST, et al: Chemokine receptor CXCR4 expression in colorectal cancer patients increases the risk for recurrence and for poor survival. J Clin Oncol 23: 2744-2753, 2005.

35. Koizumi K, Hojo S, Akashi T, Yasumoto K and Saiki I: Chemokine receptors in cancer metastasis and cancer cellderived chemokines in host immune response. Cancer Sci 98: 1652-1658, 2007.

36. Kucia M, Reca R, Miekus K, et al: Trafficking of normal stem cells and metastasis of cancer stem cells involve similar mechanisms: pivotal role of the SDF-1-CXCR4 axis. Stem Cells 23: 879-894, 2005

37. Zhang SS, Han ZP, Jing YY, et al: $\mathrm{CD} 133^{+} \mathrm{CXCR} 4^{+}$colon cancer cells exhibit metastatic potential and predict poor prognosis of patients. BMC Med 10: 85, 2012. 